\title{
Teste de padrão harmônico em escuta dicótica com dígitos - TDDH
}

\section{Harmonic pattern dichotic digits test (TDDH)}

\author{
Alejandro Arroyo Ríos ${ }^{1}$, Alessandra Giannico de Rezende ${ }^{2}$, Sandra Maria Pela ${ }^{3}$, Karin Zazo Ortiz ${ }^{4}$, \\ Liliane Desgualdo Pereira ${ }^{5}$
}

\begin{abstract}
RESUMO
Objetivo: O presente estudo teve como objetivos a elaboração de um teste de processamento auditivo com estímulos de fala cantada em tarefa dicótica, a caracterização do desempenho de ouvintes neste teste; e a comparação deste desempenho com o observado no Teste Dicótico de Dígitos. Métodos: Elaborou-se o teste com estímulos de fala cantada que foi denominado de Teste de Padrão Harmônico em Escuta Dicótica com Dígitos, composto de uma etapa de integração biaural e duas de separação biaural (direita/ esquerda), cada uma delas com 20 itens de quatro estímulos. Este foi aplicado em 40 indivíduos de ambos os sexos, falantes do português brasileiro e com idades entre 19 e 52 anos. Resultados: Os resultados foram comparados com os obtidos na aplicação do teste dicótico com estímulos de fala convencional nestes mesmos indivíduos. Os indivíduos apresentaram desempenho de aproximadamente $99 \%$ de acertos durante a aplicação do teste com estímulos de fala cantada em todas as etapas, sendo este desempenho superior ao observado no teste convencional com estímulos de fala, nas etapas de separação biaural. Conclusão: Desta forma, acredita-se que o teste elaborado no presente estudo mostrou-se aplicável em indivíduos ouvintes, sendo que o uso de estímulos de fala cantada pode modificar o desempenho nas tarefas de escuta dicótica em separação biaural, quando comparado com testes convencionais com estímulos verbais.
\end{abstract}

Descritores: Audição; Testes auditivos/métodos; Estimulação auditiva/utilização; Percepção auditiva

\section{INTRODUÇÃO}

Assim como outros sistemas sensoriais, o da audição é ordenado hierarquicamente, ou seja, por meio de uma seqüência de estações de processamento neural, que vão da periferia até o córtex auditivo. Células distintas do sistema auditivo no cérebro respondem melhor às freqüências específicas; células vizinhas têm curvas de afinação que se sobrepõem, sem nenhum ponto descoberto, formando um "mapa de frequiência" na superfície do córtex auditivo. O sistema auditivo tem uma organização tonotópica e existe uma projeção assimétrica no tálamo que permite realizar

Trabalho realizado no Departamento de Fonoaudiologia da Universidade Federal de São Paulo - UNIFESP - São Paulo (SP), Brasil, com apoio do CNPq.

(1) Doutor, Professor do Departamento de Especialidades Médicas da Escola de Medicina da Universidad de la Frontera, Chile.

(2) Pós-graduanda em Ciências pela Universidade Federal de São Paulo UNIFESP - São Paulo (SP), Brasil.

(3) Pós-graduanda em Ciências pela Universidade Federal de São Paulo UNIFESP - São Paulo (SP), Brasil.

(4) Doutora, Professor Adjunto do Departamento de Fonoaudiologia da Universidade Federal de São Paulo - UNIFESP - São Paulo (SP), Brasil.

(5) Livre Docente, Professora Associada do Departamento de Fonoaudiologia da Universidade Federal de São Paulo - UNIFESP - São Paulo (SP), Brasil.

Endereço para correspondência: Alessandra Giannico de Rezende. R. Oscar Freire, 1364/81, São Paulo-SP, CEP 05409010.E-mail: alegrezende@uol.com.br Recebido em: 18/12/2006; Aceito em: 12/11/2007 operações que não seriam possíveis se esta fosse simétrica, sendo que a cóclea possui uma correlação fisiológica do lugar onde cada frequiência está representada ${ }^{(1-2)}$. Este arranjo anatômico tem como conseqüência o conceito de que neurônios de um lugar específico têm uma característica comum de freqüência à qual aquele neurônio é mais sensível. Desta forma, para processar a informação via sentido da audição, os sons têm que ser detectados e interpretados, isto é, os estímulos acústicos são recebidos pelo sistema periférico e codificados neuralmente e, portanto, transformados em representações internas que serão analisadas e integradas pelo Sistema Auditivo Central.

Ao se procurar definições do termo Processamento Auditivo na literatura especializada encontra-se, de forma global, referências sobre mecanismos que o sistema auditivo periférico e central utiliza para analisar e organizar as informações em resposta a um estímulo acústico ${ }^{(1,3-5)}$ indicando que estes mecanismos estão previstos, tanto para o processamento com sons verbais quanto não-verbais e que muitos processos neurocognitivos estão envolvidos nas tarefas de processamento auditivo $^{(6)}$.

Este termo refere-se à integração destes mecanismos, na atividade do processamento do sinal acústico que, por sua própria natureza, pode ser interpretado como um conjunto de componentes das habilidades auditivas, algumas das quais se relacionam preferencialmente ao processamento musical. $\mathrm{O}$ hemisfério direito é dominante para a percepção da música ${ }^{(2,7-9)}$ 
e, desta forma, provavelmente o responsável pelo reconhecimento global do padrão gestáltico, das relações espaciais e dos aspectos da interação inter-hemisférica através do corpo caloso, também envolvido neste tipo de atividade ${ }^{(10-11)}$.

Há um consenso na literatura especializada em se utilizar testes de escuta dicótica para avaliar o processamento neurológico de informações auditivas recebidas pelo indivíduo. Esse tipo de tarefa possibilita estudar os efeitos da lateralidade quando a modalidade do estímulo é auditiva, ou seja, verificar a assimetria interaural para sons verbais e não verbais $^{(12-14)}$. Dentre estes testes, tem-se o Teste Dicótico de Dígitos que avalia a habilidade auditiva de figura-fundo para sons verbais, por meio da tarefa de escuta dicótica / integração binaural. Os estímulos verbais do teste são constituídos por vocábulos que representam dígitos apresentados em tarefa dicótica, utilizados na rotina de avaliação auditiva comportamental em populações com ou sem risco de alterações do processamento auditivo ${ }^{(15-21)}$. Sabe-se que a identificação correta dos estímulos recebidos pela orelha direita indica integridade neurobiológica, incluindo a comunicação inter-hemisférica em nível de corpo caloso e resultados alterados em ambas as orelhas sugerem alterações no hemisfério esquerdo ${ }^{(3)}$.

A música assim como a linguagem é um meio de comunicação. Os sons musicais são essencialmente baseados em processamento neurológico de informações acústicas, e dependem da identificação e do tipo de associações emocionais com experiências anteriores. Sendo assim, cabe destacar que tanto os mecanismos neurais inatos (operações de processamento primário), a vivência cultural (mensagens armazenadas e operações de processamento aprendidas), quanto às emoções devem determinar a resposta comportamental aos estímulos musicais.

As primeiras informações sobre o processamento auditivo musical no cérebro foram estudadas em indivíduos com alterações neurológicas e auditivas, entre eles em famosos compositores, como Maurice Ravel ${ }^{(22)}$. Sabe-se que o cérebro poderia não ter um centro específico para o processamento auditivo musical, sendo que a relação cérebro-música englobaria uma série de processos muito elaborados.

Com relação aos estímulos musicais, há um consenso de que, quando o hemisfério direito recepciona os sons musicais vindos pela orelha esquerda, o córtex auditivo provoca conexões em direção a outras regiões hemisféricas, como as áreas corticais de relação e de interpretação. O processamento auditivo da música é modulado pelo sistema límbico e paralímbico, zona cortical profunda que coordena diversas variáveis emocionais e de relação inter-hemisférica cerebral ${ }^{(23)}$.

Como a audição tem sua influência tanto no processamento da linguagem como da música, estas não podem ser consideradas como entidades isoladas, precisando ser estudadas em suas inter-relações, seus diferentes componentes ou níveis de processamento ${ }^{(8,24-25)}$.

Utilizar sons musicais associados ao estimulo verbal poderia facilitar o reconhecimento destes e ser útil na reabilitação auditiva e de linguagem de indivíduos com alteração da comunicação, considerando-se as habilidades de discriminação de sons em escuta dicótica, de agrupamento de com- ponentes do sinal acústico em figura-fundo e a identificação deste som em termos verbais.

Com base nessa idéia o Teste Dicótico de Dígitos, recomendado na clínica para crianças desde cinco anos de idade, por sua facilidade de aplicação e de resposta, pareceu ser apropriado para o desenvolvimento de um procedimento deste tipo. E assim, estudou-se a possibilidade de associar pistas musicais aos estímulos verbais desse teste.

Desta forma, os objetivos deste estudo foram: elaborar um teste de processamento auditivo com estímulos musicais, utilizando-se fala cantada e verificar o desempenho de adultos ouvintes normais neste teste, além de comparar com o desempenho no teste auditivo convencional denominado de Teste Dicótico de Dígitos.

A hipótese deste estudo é a de que pistas musicais melhoram o reconhecimento de palavras familiares em escuta dicótica. Essa hipótese foi levantada, pensando-se em uma contribuição maior do hemisfério direito no processamento de fala em tarefa dicótica.

Este conhecimento poderá auxiliar o direcionamento da reabilitação auditivo-verbal em indivíduos com alterações da linguagem.

\section{MÉTODOS}

Esta pesquisa foi realizada no Ambulatório da Disciplina de Distúrbios da Audição do Departamento de Fonoaudiologia da Universidade Federal de São Paulo, recebendo aprovação do Comitê de Ética e Pesquisa da UNIFESP/HSP sob o número 0056/04. Todos os participantes do estudo assinaram o Termo de Consentimento Livre e Esclarecido antes do início dos procedimentos.

Este é um estudo observacional, com levantamento populacional amostral, em que participaram 40 indivíduos adultos ouvintes, também denominados ouvintes típicos, falantes de português brasileiro, residentes na cidade de São Paulo. Os sujeitos foram voluntários recrutados na UNIFESP/ campus São Paulo. Vale ressaltar que não tinham contato prévio com os testes aplicados no presente estudo. Do total de indivíduos, 27 eram do sexo feminino e 13 do sexo masculino, com faixa etária variando entre 19 e 52 anos. A escolha da faixa etária, bem como o pré-requisito de serem ouvintes normais se deu pela facilidade de aplicação e praticidade do modo de resposta dos referidos testes. Todos foram submetidos à avaliação audiológica convencional descartando-se, desta forma, evidências de problemas auditivos. Os padrões de normalidade utilizados foram os descritos na literatura e comumente usados na rotina de atendimento auditivo ${ }^{(26-27)}$, ou seja, limiares de audibilidade para tons puros até $20 \mathrm{~dB}$ NA nas freqüências de $250 \mathrm{~Hz}$ a $8000 \mathrm{~Hz}$ e índice de reconhecimento da fala igual ou superior a $92 \%$ de acertos, curva timpanométrica sem evidências de alterações de condução do som e presença de reflexos acústicos.

\section{Descrição da elaboração do teste}

O teste elaborado no presente estudo foi denominado de Teste de Padrão Harmônico em Escuta Dicótica com Dígitos 
(TDDH). Sua elaboração foi baseada no Teste Dicótico de Dígitos $^{(28-29)}$, utilizando-se os dígitos "quatro", "cinco", "sete", "oito" e "nove", sendo harmônicos e correspondentes ao tema de "Musikalisches Ofter" do compositor Johann Sebastian Bach. Cada seqüência com quatro estímulos de fala cantada corresponde a um item. Esta seqüência harmônica se fundamenta na teoria de que o ser humano possui audição tonotópica ${ }^{(1-2)}$, ou seja, as diferentes frequiências são processadas em locais distintos do sistema auditivo, desde a periferia até o córtex. Outra característica é a de que as notas utilizadas foram cantadas por uma voz mezzo-soprano, ficando dentro do âmbito vocal de um indivíduo comum, a fim de evitar problemas que se apresentam pelos registros musicais, permanecendo audíveis por, aproximadamente, quatro segundos.

O teste foi gravado em estúdio, na forma de compact disc - CD, devendo ser apresentado à mesma intensidade em ambas as orelhas, a um nível de sensação de $50 \mathrm{~dB}$ NS levando-se em consideração a sensibilidade auditiva da melhor orelha (média dos limiares de $500 \mathrm{~Hz}, 1000 \mathrm{~Hz}$ e 2000 $\mathrm{Hz}$ por via aérea) utilizando-se fones TDH-39. Apresentouse um tom puro de $1000 \mathrm{~Hz}$ a $60 \mathrm{~dB}$, no inicio do CD, como referência para a calibração da gravação do audiômetro de dois canais. Houve o cuidado de verificar e manter a mesma calibração do CD, antes de iniciar cada uma das apresentações via audiômetro, em cada sujeito avaliado. O teste completo teve a duração de aproximadamente 30 minutos.

Os estímulos foram apresentados aos pares e de forma dicótica. Antes de cada item foi gravada a palavra repita, em diferentes tonalidades com a finalidade de garantir a atenção do indivíduo avaliado.

Este teste constou de três etapas, cada uma composta por 20 itens, totalizando 80 estímulos de fala cantada. Estas etapas foram a de Atenção Livre/integração biaural, Atenção Direcionada à Direita/separação biaural, e Atenção Direcionada à Esquerda/separação biaural. Na etapa de Atenção Livre/integração biaural os quatro estímulos de fala cantada ouvidos deveriam ser repetidos oralmente. Já nas etapas de Atenção Direcionada, deveriam ser repetidos de forma oral apenas os estímulos correspondentes ao lado que estava sendo avaliado no momento, considerando-se como erro a omissão dos referidos estímulos ou a identificação dos apresentados na orelha oposta.

As respostas corretas foram registradas com um círculo ao redor da palavra identificada. Em caso de erro, omissão ou substituição de uma palavra, este foi assinalado com um traço correspondente e a resposta foi anotada ao lado do estímulo apresentado. Para análise do resultado, cada estímulo de fala cantada identificado incorretamente equivaleu a um erro. Os resultados foram apresentados em porcentagem de acertos. Cada estímulo de fala cantada identificado corretamente correspondeu a $2,5 \%$ e foi computado por orelha. Assim, o total de acertos de $100 \%$ da orelha direita correspondeu à identificação correta dos 40 estímulos de fala cantada recebidos por esta orelha. O mesmo foi feito na analise da orelha esquerda desta etapa. Na etapa de escuta direcionada, calculamos a porcentagem de acertos, considerando os estímulos apresentados na orelha solicitada (40 dígitos harmônicos). Para esse cálculo, cada estímulo identificado incor- retamente também equivaleu a um erro.

Os estímulos deste teste novo são constituídos por dígitos harmônicos para separação didática da denominação dos estímulos verbais do teste dicótico de dígitos convencional, que são os mesmos. O que diferencia os estímulos de cada teste é o acréscimo da pista musical, isto é, fala cantada.

Após a fase de elaboração do teste, iniciaram-se as avaliações de cada indivíduo, separadamente, em cabina acústica e com audiômetro de dois canais.

Estes foram submetidos, inicialmente, ao Teste Dicótico de Dígitos segundo o procedimento proposto na literatura ${ }^{28-}$ ${ }^{29)}$. Este teste foi selecionado por ser de fácil aplicação e constituído de estímulos verbais de alta previsibilidade, possibilitando uma boa margem de acertos em indivíduos com mais de cinco anos. Os resultados foram anotados em uma folha de resposta própria.

Em seguida, os indivíduos foram submetidos ao Teste de Padrão Harmônico em Escuta Dicótica com Dígitos. As respostas dos indivíduos foram transcritas em uma folha de registros. Assim, verificou-se o número de estímulos que o indivíduo identificou corretamente, denominado de "acertos", e o número de estímulos não identificados corretamente, chamado de "erros". Foi registrada na folha própria toda resposta diferente da correta.

Antes da aplicação dos testes, foi realizado um treino demonstrativo para que o indivíduo compreendesse bem a tarefa a ser realizada. As avaliações duraram aproximadamente 60 minutos.

Após as avaliações, foram computados os acertos de cada teste, por indivíduo, verificando o desempenho no teste elaborado para o estudo e comparando-se este desempenho com o apresentado no Teste Dicótico de Dígitos.

Foi realizado um estudo estatístico dos resultados por meio do Teste de Fisher, do Teste de Wilcoxon e apresentação em Box-plot ${ }^{(30)}$. O nível de significância adotado foi de $5 \%$.

\section{RESULTADOS}

Na Tabela 1 estão demonstrados os resultados, em porcentagem de acertos por orelha, nas etapas de atenção livre e direcionada obtidos por meio da aplicação do novo teste denominado de TDDH em 40 adultos ouvintes saudáveis.

Com relação ao desempenho dos 40 indivíduos, selecionados para a aplicação do TDDH, pôde-se observar que 39 $(97,5 \%)$ apresentaram porcentagem de acertos superior a $95 \%$ por orelha na etapa de atenção livre. Já nas etapas de atenção direcionada à direita e à esquerda os 40 (100\%) indivíduos pesquisados tiveram desempenho superior a $95 \%$ de acertos. Ao comparar-se o número de acertos no TDDH em atenção livre para orelha direita e esquerda, verificou-se a não existência de diferença estatisticamente significante (Tabela 1).

Para efeitos de comparação entre os dados obtidos com a aplicação do novo teste (TDDH) e do teste convencional (TDD) apresenta-se na Tabela 2, os resultados em porcentagem de acertos, por orelha na etapa de atenção livre e, na Tabela 3 para a etapa de atenção direcionada e os p-valores calculados. Ilustram-se esses resultados na Figura 1 (atenção livre) e Figura 2 (atenção direcionada). 
Tabela 1. Número de indivíduos com relação à porcentagem de acertos obtidos no TDDH $(\mathrm{N}=40)$

\begin{tabular}{|c|c|c|c|c|c|c|c|c|}
\hline \multirow{3}{*}{ Acertos (\%) } & \multicolumn{8}{|c|}{ TDDH } \\
\hline & \multicolumn{2}{|c|}{ OD } & \multicolumn{2}{|c|}{ OE } & \multicolumn{2}{|c|}{ OD } & \multicolumn{2}{|c|}{ OE } \\
\hline & $\mathrm{N}$ & $\%$ & $\mathrm{~N}$ & $\%$ & $\mathrm{~N}$ & $\%$ & $\mathrm{~N}$ & $\%$ \\
\hline 100,0 & 37 & 92,5 & 37 & 92,5 & 34 & 85 & 36 & 90 \\
\hline Total & 40 & 100,0 & 40 & 100,0 & 40 & 100,0 & 40 & 100,0 \\
\hline
\end{tabular}

Teste Exato de Fisher

$p=0,6890$

Legenda: TDDH: Teste de Padrão Harmônico em Escuta Dicótica com Dígitos; OD: Orelha direita. OE: Orelha esquerda N: número absoluto de indivíduos; $\%$ : porcentagem

Tabela 2. Número de indivíduos com relação à porcentagem de acertos obtidos na Etapa de Atenção Livre dos testes TDDH e TDD ( $\mathrm{N}=40$ )

\begin{tabular}{|c|c|c|c|c|c|c|c|c|}
\hline \multirow{4}{*}{ Acertos (\%) } & \multicolumn{4}{|c|}{ TDDH } & \multicolumn{4}{|c|}{ TDD } \\
\hline & \multicolumn{4}{|c|}{ Atenção Livre } & \multicolumn{4}{|c|}{ Atenção Livre } \\
\hline & \multicolumn{2}{|c|}{ OD } & \multicolumn{2}{|c|}{ OE } & \multicolumn{2}{|c|}{ OD } & \multicolumn{2}{|c|}{ OE } \\
\hline & $\mathrm{N}$ & $\%$ & $\mathrm{~N}$ & $\%$ & $\mathrm{~N}$ & $\%$ & $\mathrm{~N}$ & $\%$ \\
\hline 100,0 & 37 & 92,5 & 37 & 92,5 & 16 & 40 & 18 & 45 \\
\hline $99-95$ & 2 & 5 & 3 & 7,5 & 24 & 60 & 21 & 52,5 \\
\hline$\leq 95$ & 1 & 2,5 & 0 & 0 & 0 & 0 & 1 & 2,5 \\
\hline Total & 40 & 100,0 & 40 & 100,0 & 40 & 100,0 & 40 & 100,0 \\
\hline \multicolumn{9}{|c|}{ Teste de Wilcoxon } \\
\hline \multicolumn{9}{|c|}{ TDDH $x$ TDD } \\
\hline \multicolumn{9}{|c|}{ Etapa de Atenção Livre: } \\
\hline \multicolumn{9}{|c|}{ Orelha esquerda $p=0,002^{*} \quad$ Orelha direita $p=0,002^{*}$} \\
\hline
\end{tabular}

Legenda: TDDH: Teste de Padrão Harmônico em Escuta Dicótica com Dígitos; TDD: Teste Dicótico de Dígitos; OD: Orelha direita; OE: Orelha esquerda; N: número absoluto de indivíduos; \%: porcentagem; * estatisticamente significante

Tabela 3. Número de indivíduos com relação à porcentagem de acertos obtidos na Etapa de Atenção Direcionada à Direita e à Esquerda dos testes TDDH e TDD $(\mathrm{N}=40)$

\begin{tabular}{|c|c|c|c|c|c|c|c|c|}
\hline \multirow{4}{*}{ Acertos (\%) } & \multicolumn{4}{|c|}{ TDDH } & \multicolumn{4}{|c|}{ TDD } \\
\hline & \multicolumn{4}{|c|}{ Atenção Direcionada } & \multicolumn{4}{|c|}{ Atenção Direcionada } \\
\hline & \multicolumn{2}{|c|}{ OD } & \multicolumn{2}{|c|}{ OE } & \multicolumn{2}{|c|}{ OD } & \multicolumn{2}{|c|}{ OE } \\
\hline & $\mathrm{N}$ & $\%$ & $\mathrm{~N}$ & $\%$ & $\mathrm{~N}$ & $\%$ & $\mathrm{~N}$ & $\%$ \\
\hline 100,0 & 34 & 85 & 36 & 90 & 11 & 27,5 & 10 & 25 \\
\hline $99-95$ & 6 & 15 & 4 & 10 & 27 & 67,5 & 25 & 62,5 \\
\hline$\leq 95$ & 0 & 0 & 0 & 0 & 2 & 5 & 5 & 12,5 \\
\hline Total & 40 & 100,0 & 40 & 100,0 & 40 & 100,0 & 40 & 100,0 \\
\hline \multicolumn{9}{|c|}{ Teste Exato de Fisher } \\
\hline \multicolumn{9}{|c|}{ TDDH $x$ TDD } \\
\hline \multicolumn{9}{|c|}{ Etapa de escuta Direcionada } \\
\hline \multicolumn{9}{|c|}{ Orelha direita $=p=0,0001^{*}$} \\
\hline \multicolumn{9}{|c|}{ Orelha esquerda $p=0,0001^{*}$} \\
\hline
\end{tabular}

Legenda: TDDH: Teste de Padrão Harmônico em Escuta Dicótica com Dígitos; TDD: Teste Dicótico de Dígitos; OD: Orelha direita; OE: Orelha esquerda; N: número absoluto de indivíduos; \%: porcentagem; * estatisticamente significante 


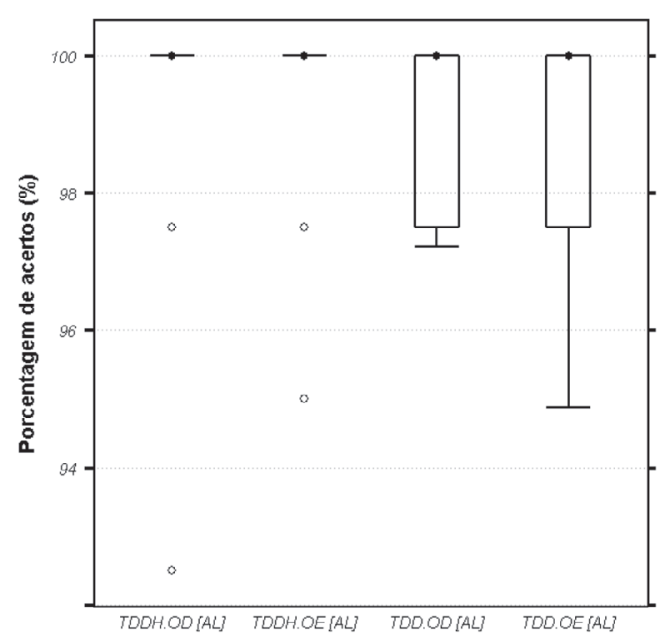

Legenda: TDDH: Teste de Padrão Harmônico em Escuta Dicótica com Dígitos; OD: Orelha direita; OE: Orelha esquerda; AL: Atenção Livre

Figura 1. Box-plot dos valores em porcentagem de acertos no TDD e no TDDH na etapa de atenção livre.

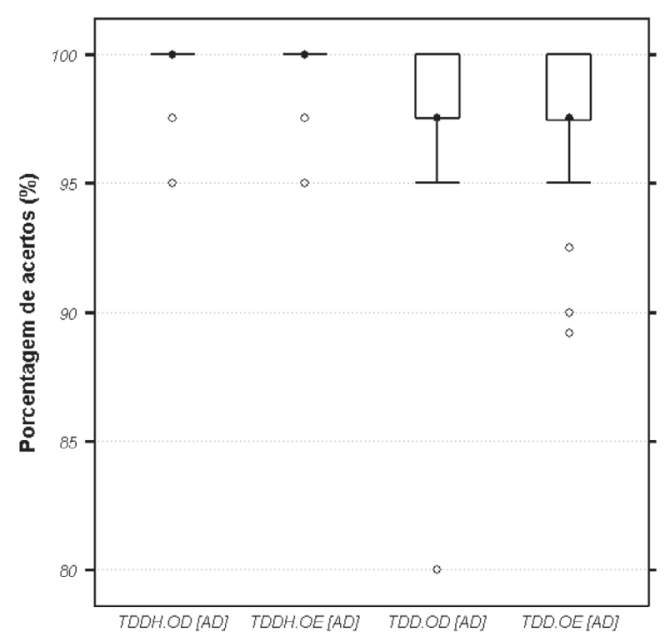

Legenda: TDDH: Teste de Padrão Harmônico em Escuta Dicótica com Dígitos; OD: Orelha direita; OE: Orelha esquerda; AD: Atenção Direcionada

Figura 2. Box-plot dos valores em porcentagem de acertos no TDD e no TDDH na etapa de atenção direcionada (AD) para orelha direita (OD) e para esquerda $(\mathrm{OE})$.

Na comparação entre os testes TDDH e TDD ${ }^{(20-23)}$, durante a etapa de atenção livre (Tabela 2) e de escuta direcionada (Tabela 3), verificaram-se diferenças estatisticamente significantes nas respostas de ambas orelhas, podendo-se observar um número de acertos significantemente maior no TDDH em relação ao TDD. Assim, verificou-se que os acertos para o teste com estímulos de fala cantada foram maiores do que para o teste convencional.

\section{DISCUSSÃO}

Neste estudo, o Teste de Reconhecimento de Padrão Har- mônico em Escuta Dicótica com Dígitos - TDDH foi elaborado para avaliar o desempenho em escuta dicótica: integração e separação biaural. O procedimento possibilita medir a habilidade de agrupar componentes do sinal acústico em figura-fundo, nas etapas de atenção livre (integração biaural) e atenção direcionada (separação biaural).

O modelo citado na literatura ${ }^{(3-6)}$ utilizado para explicar a função do sistema nervoso auditivo central, na percepção dos estímulos apresentados dicoticamente, pode ser aplicado no entendimento deste comportamento. Desta forma, pode-se levantar a hipótese de que a superioridade da orelha direita não é absoluta quando se utilizam estímulos verbais com redundâncias de pistas acústicas. Novos estudos devem ser feitos nesta direção.

Uma hipótese para o desempenho elevado encontrado no teste de processamento auditivo, com estímulos de fala cantada seria a de que cada nota musical (estímulo físico) é capaz de provocar imagens mentais (representação interna) de uma sequiência harmônica completa ${ }^{(2,8-9,25)}$. Assim, acredita-se que tenha ocorrido um fenômeno de ilusão musical, visto que os estímulos apresentados atuam de forma harmônica e melódica. Parece que houve maior contribuição do hemisfério direito, no processamento neurológico desta tarefa dicótica com fala cantada. Outra hipótese para compreender a supremacia da dimensão harmônica do som demonstrada no TDDH seria a organização tonotópica de frequiências no sistema auditivo.

Também se sabe que há o envolvimento dos dois hemisférios cerebrais no desempenho do teste, visto que a melodia e a harmonia estão vinculadas ao hemisfério direito ${ }^{(2,7-9,21-}$ ${ }^{22,24)}$, enquanto a produção e a compreensão da linguagem falada e do ritmo musical são tarefas do hemisfério esquer$\mathrm{do}^{(20)}$. Assim, acredita-se que, ao se utilizar procedimentos elaborados para a avaliação do processamento auditivo com estímulos estímulo de fala cantada, ambos os hemisférios cerebrais participam mais ativamente durante a aplicação do teste $^{(10-11)}$, o que poderia ajudar a explicar o melhor desempenho dos indivíduos.

Ao se pensar nisto, pode-se ver a música como produto do desenvolvimento das várias habilidades envolvidas no processamento auditivo, acreditando-se que ela favoreça o desenvolvimento de outras habilidades ${ }^{(8,25,27)}$, apresentando grande potencial para ser utilizada como instrumento preventivo e/ou terapêutico nos possíveis distúrbios de processamento auditivo, de linguagem e de aprendizagem.

Para que este novo teste seja utilizado na prática clínica, muitos estudos ainda serão necessários, considerando as populações de crianças, adolescentes, idosos e de indivíduos com lesão cerebral e com perda auditiva.

\section{CONCLUSÕES}

Com base nos achados, acredita-se que o teste dicótico com fala cantada, elaborado e denominado de Teste de Reconhecimento de Padrão Harmônico em Escuta Dicótica com Dígitos (TDDH), mostrou ser de fácil aplicação em adultos ouvintes e melhorou o desempenho desta amostra populacional, quando comparado ao teste convencional com os mesmos estímulos de fala denominado de Teste Dicotico de Dígitos. 


\begin{abstract}
Purpose: The aims of this study are the development of an auditory processing test with musical stimuli in dichotic tasks, the characterization of the performance of normal hearing subjects and the comparison between the results and those in the Digits Dichotic Test (TDD). Methods: The auditory processing test with musical stimuli was elaborated with harmonic digits. The test was denominated Harmonic Pattern Dichotic Digits Test (TDDH) and comprised biaural integration and two stages of biaural separation (left/right), each one with 20 items made up of four harmonic digits. It was applied in 40 individuals of both sexes. They were all adults, their ages ranged from 19 to 52 years old and they spoke Brazilian Portuguese. Results: The results were compared to results of the TDD applied in the same individuals. The individuals had approximately $99 \%$ success in all the stages, their performance was higher than the one observed in the TDD - stages of biaural separation. Conclusion: We believe that the test elaborated for this study showed applicability in normal hearing individuals. The use of musical stimuli seems to make the performance of individuals easier than the dichotic verbal tests.
\end{abstract}

Keywords: Hearing; Hearing tests/methods; Acoustic stimulation/utilization; Auditory perception

\title{
REFERÊNCIAS
}

1. Phillips DP. Central auditory processing: a view from auditory neuroscience. Am J Otol. 1995;16(3):338-52.

2. Liégeois-Chaveul C, Giraud K, Badier JM, Marquis P, Chauvel P. Intracerebral evoked potentials in pitch perception reveal a functional asymmetry of the human auditory cortex. Ann N Y Acad Sci. 2001;930:177-32.

3. Kimura D. Functional asymmetry of the brain in dichotic listening. Cortex. 1967;3:163-78.

4. Milner B, Taylor S, Sperry RW. Lateralized suppression of dichotically presented digits after commissural section in man. Science. 1968;161(837):184-6.

5. American Speech-Language-Hearing Association (2005). (Central) Auditory processing disorders [Technical Report]. [cited 2007 July 12]. Available at http://www.asha.org/docs/pdf/TR2005-00043.pdf

6. Kimura D. Some effects of temporal-lobe damage on auditory perception. Can JPsychol. 1961;15:156-65.

7. Zatorre RJ. Neural specializations for tonal processing. Ann N Y Acad Sci. 2001;930:193-210.

8. Koelsch S, Maess B, Gunter TC, Friederici AD. Neapolitan chords activate the area of Broca. A magnetoencephalographic study. Ann N Y Acad Sci. 2001;930:420-1.

9. Griffiths TD. The neural processing of complex sounds. Ann N Y Acad Sci. 2001;930:133-42.

10. Musiek FE, Pinheiro ML, Wilson DH. Auditory pattern perception in 'split-brain' patients. Arch Otolaryngol. 1980;106(10):610-2.

11. Musiek FE, Baran JA, Pinheiro ML. Neuroaudiology cases studies. San Diego: Singular; 1994.

12. Jerger J, Martin J. Dichotic listening tests in the assessment of auditory processing disorders. Audiol Med. 2006;4(1):25-34.

13. Roup CM, Wiley TL, Wilson RH. Dichotic word recognition in young and older adults. J Am Acad Audiol. 2006;17(4):230-40; quiz 297-8. Comment in: J Am Acad Audiol. 2006;17(4):2 p following table of contents.

14. Plessen KJ, Lundervold A, Grüner R, Hammar A, Lundervold A, Peterson BS, Hugdahl K. Functional brain asymmetry, attentional modulation, and interhemispheric transfer in boys with Tourette syndrome. Neuropsychologia. 2007;45(4):767-74.

15. Moncrieff DW, Musiek FE. Interaural asymmetries revealed by dichotic listening tests in normal and dyslexic children. J Am Acad Audiol. 2002;13(8):428-37.
16. Onoda RM, Pereira LD, Guilherme A. Reconhecimento de padrão temporal e escuta dicótica em descendentes de japoneses, falantes ou não-falantes da língua japonesa. Rev Bras Otorrinolaringol. 2006;72(6):737-46.

17. Meneguello J, Leonhardt FD, Pereira LD. Processamento auditivo em indivíduos com epilepsia de lobo temporal. Rev Bras Otorrinolaringol. 2006;72(4):496-504.

18. Ziliotto KN, Santos MFC, Monteiro VG, Pradella-Hallinan M, Moreira GA, Pereira LD, et al. Avaliação do processamento auditivo em crianças com síndrome de apnéia/hipopnéia obstrutiva do sono. Rev Bras Otorrinolaringol. 2006;72(3):321-7.

19. Bamiou DE, Musiek FE, Stow I, Stevens J, Cipolotti L, Brown MM, Luxon LM. Auditory temporal processing deficits in patients with insular stroke. Neurology. 2006;67(4):614-9.

20. Bamiou DE, Campbell NG, Musiek FE, Taylor R, Chong WK, Moore A, et al. Auditory and verbal working memory deficits in a child with congenital aniridia due to PAX6 mutation. Int J Audiol. 2007;46(4):196-202.

21. Hough MS, Givens GD, Cranford JL, Downs RC. Behavioural and electrophysiological measures of auditory attention in right hemisphere brain damage. Aphasiology. 2007;21(9):831-43.

22. Pooper KR, Eccles J. O cérebro e o pensamento. Campinas: Papirus; 1992.

23. Herrero JV, Hillix WA. Hemispheric performance in detecting prosody: a competitive dichotic listening task. Percept Mot Skills. 1990;71(2):479-86.

24. Ortiz KZ, Pereira LD, Borges ACLC, Vilanova LC. Verbal and non verbal auditory processing: a comparative study. Iran Audiol. 2003;2(1):152-60.

25. Brown S. Are music and language homologues? Ann N Y Acad Sci. 2001;930:372-4.

26. Hodgson WR. Speech discrimination testing. In: Hodgson WR. Basic audiologic evaluation. Baltimore: Williams\& Wilkins; c1980. p.137-75.

27. Pereira LD, Gentile C, Osterne FJV, Borges ACLC, Fukuda Y. Considerações preliminares no estudo do teste de fala com ruído em indivíduos normais. Acta AWHO. 1992;11(3):119-22.

28. Musiek FE. Assessment of central auditory dysfunction: the dichotic digit test revisited. Ear Hear. 1983;4(2):79-83.

29. Santos MFC. Teste de escuta dicótica com dígitos [tese]. São Paulo: Universidade Federal de São Paulo. Escola Paulista de Medicina; 1996.

30. Vieira S. Bioestatística: tópicos avançados. 2a ed. rev. e atual. Rio de Janeiro: Campus; 2004. 216 p. 